\title{
Atomic-Resolution X-ray Analysis in Aberration-Corrected Scanning Transmission Electron Microscopes: Current Limits and Challenges toward Quantification
}

\author{
M. Watanabe
}

Dept of Materials Science and Engineering, Lehigh University, Bethlehem. PA 18015.

X-ray analysis has been widely performed as one of the most robust characterization approaches in scanning transmission electron microscopes (STEMs) for $\sim 40$ years because of its simple operation and interpretation nature. However, availability of X-ray signals is very limited due to a small analyzed volume, physically restricted signal-generation and poor signal-detection configurations of detectors. These limitations are now compensated in some degree by using the latest aberration-corrected STEMs in combination with the large solid-angle silicon drift detectors (SDDs) $[1,2]$. It is now possible to acquire atomic-resolution X-ray maps using the latest instruments such as an aberration-corrected STEM JEOL JEM-ARM200CF equipped with a large solid-angle SDD. In this study, possibilities and limitations for quantification of atomic-resolution X-ray analysis will be explored.

Figure 1 shows a set of atomic resolution X-ray map acquired from a [ 100]-projected GaAs specimen using the aberration-corrected STEM JEM- ARM200CF operated at $200 \mathrm{kV}$. In the [100]-projection of $\mathrm{Ga} \mathrm{As}$, Ga and As layers are alternatively separated. Unfortunately, this configuration may not appear by HAADF-STEM imaging (Fig. 1a) unless a very thin specimen is observed, because the difference in the atomic number is only two between Ga (31) and As (33). As shown in the elemental maps of (b) Ga and (c) As with (d) their color overlay, separated layers of Ga and d As can be observed. Quantification of these maps were carried out by the $\zeta$-factor method [3], and compositions of (e) Ga and (f) As were determined with (g) thickness. Although the Ga and As layers seem well separated in elemental maps, the compositions do not reach to 0 or 100 at $\%$ in corresponding atomic layers. The maximum and minimum values of measured compositions are $\sim 70$ and 30 at $\%$. According to the thickness map determined from measured X-ray intensities by the $\zeta$-factor method, there are relatively large variations between on-column and off-column regions: $\sim 60$ and $30 \mathrm{~nm}$ at on- and off-column regions, respectively.

In order to investigate the composition variation of on- and off-atomic-column points, X-ray maps were measured from the [100]-projected GaAs at different thickness regions and quantified them. The quantified $\mathrm{Ga}$ compositions extracted at the Ga columns, As columns and off-columns are plotted against the specimen thickness in Fig. 2. From this plot, it is evident that (i) the compositions at both atomic columns do not reach 100:0 at \% even in the thinnest region but come close to the average composition (50:50 at $\%$ ) with an increase of the specimen thickness, (ii) the compositions at off-column positions exhibit the average composition (50:50 at\%) and (iii) the thickness values at off-column positions are lower than those at atomic column positions. This thickness enhancement at the atomic-column positions indicates that abnormal X-ray emission occurs due to channeling [4]. The map was obtained in highly symmetric zone axis, in which the incident beam propagation is influenced by the atomic arrangement, i.e. the incident electrons are channeled and dechannneled especially at the atomic columns [5 ]. The channeling/dechanneling behaviours can be seen by simulating the wave function of incident beam propagating the specimen. The wave functions at $\mathrm{Ga}$ and off columns, simulated by xHREM multislice code [6], are shown in Fig. 3. Whereas the incident beam tends to remain the atomic column, the probe at the off-column position spreads more toward neighbour columns For quantification of the atomic resolution X-ray maps, the probe propagation needs to be considered. 


\section{References}

[1] H.S. von Harrach, et al., Microsc. Microana. 15 (2009), Suppl. 2, 208.

[2] I. Ohnishi, et al, Microsc. Microana. 17 (2011), Suppl. 2, 22..

[3] M. Watanabe and D.B. Williams, J. Microsc. 221 (2006), 89.

[4] J.F. Bullock et al. Microscopy in Semiconductor Materials 1985, 405 (1985). .

[5] B.D. Forbes et al. Allen, Phys. Rev. B 86, 024108 (2012).

[6] K. Ishizuka., J. Electron Microsc. 50 (2012), 291.

[7] The author wishes to acknowledge financial supp ort from the NSF through grants DMR-0804528 and DMR-1040229.

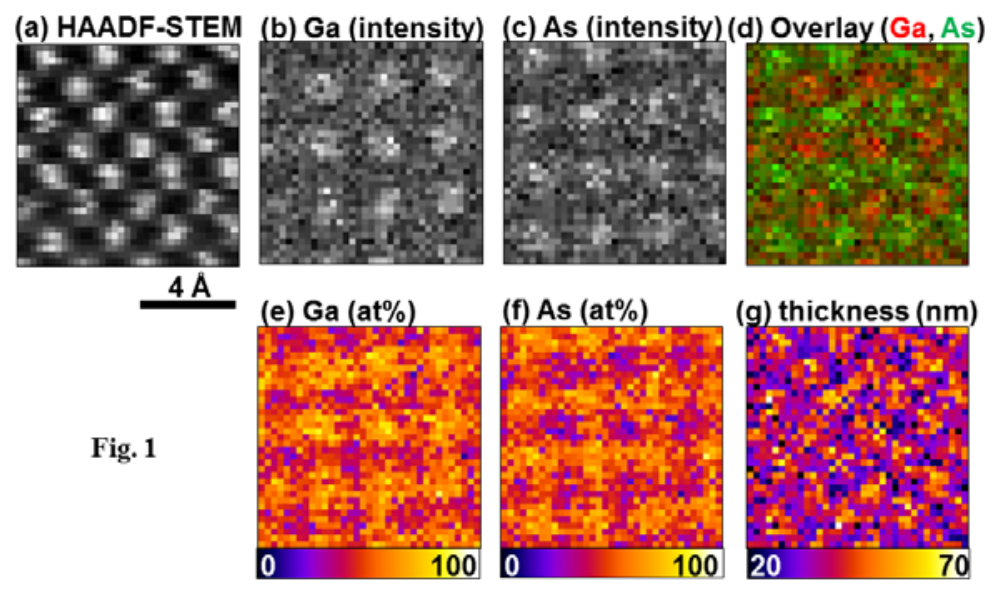

Figure 1: A set of quantitative X-ray maps from a [100]-projected GaAs specimen: (a) HAADF-STEM image, (b) Ga K intensity, (c) As K intensity, (d) color overlay of Ga K (red) and As K (green), (e) Ga composition, (f) As composition and (g) thickness.

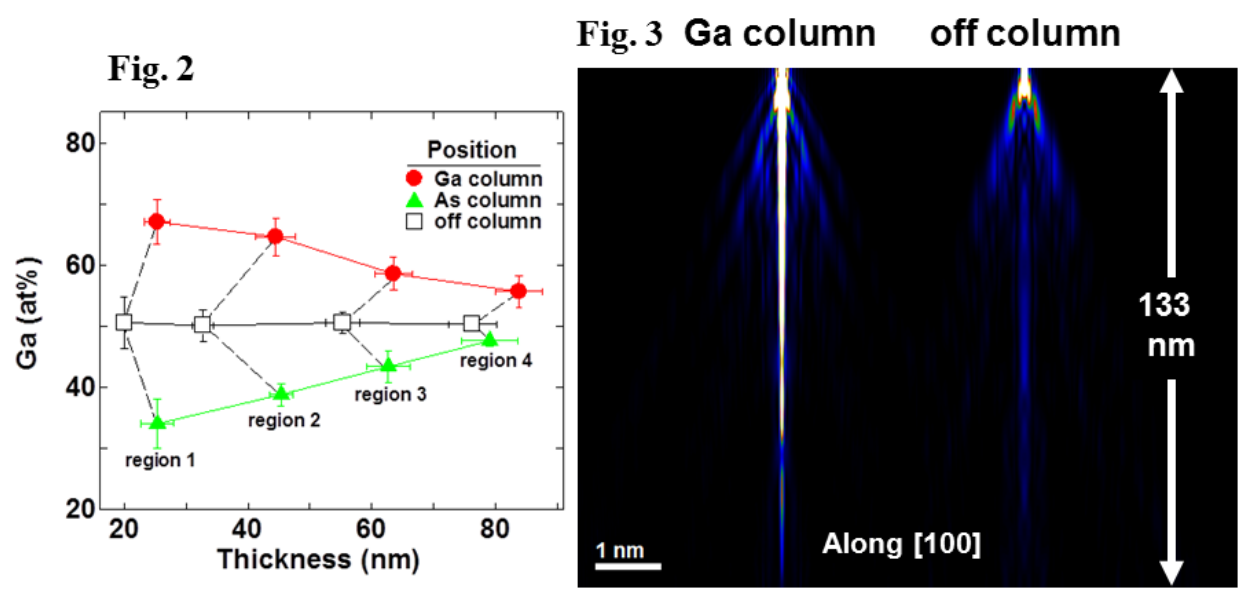

Figure 2: Ga composition extracted from $\mathrm{Ga}$, As and off column positions in atomic-resolution X-ray maps of [001]-projected GaAs, plotted against the specimen thickness.

Figure 3: Simulated wave functions at Ga and off-column positions of [001]-projected GaAs. 